\title{
Robust time-domain migration velocity analysis methods for initial-model building in a full waveform tomography workflow
}

\author{
H. B. Santos (CEP/UNICAMP \& INCT-GP), J. Schleicher (IMECC/UNICAMP \& INCT-GP), A. Novais (IMECC/UNICAMP \& \\ INCT-GP), A. Kurzmann (KIT), and T. Bohlen (KIT)
}

\begin{abstract}
Copyright 2016, SBGf - Sociedade Brasileira de Geofísica.
Este texto foi preparado para a apresentação no Simpósio Brasileiro de Geofísica, Ouro Preto, 25 a 27 de outubro de 2016. Seu conteúdo foi revisado pelo Comitê Técnico do VII SimBGf, mas não necessariamente representa a opinião da SBGf ou de seus associados. É proibida a reprodução total ou parcial deste material para propósitos comerciais sem prévia autorização da SBGt.
\end{abstract}

\begin{abstract}
Full-waveform tomography (FWT) is notorious for its strong dependence on the initial model. We present a workflow for the construction of initial velocitymodels for FWT methods consisting of automatic timemigration velocity analysis by means of double multistack migration, followed by time-to-depth conversion by image-ray wavefront propagation. Evaluation of the converted velocity model as an initial velocity model in an acoustic FWT process indicates the potential of using a combination of these methods to achieve a fully automatic tool for initial-model building in an FWT workflow. Our tests on a modified version of the Marmousi-2 model have shown that correct background velocity information can be successfully extracted from automatic time-domain migration velocity analysis even in media where timemigration cannot provide satisfactory seismic images.
\end{abstract}

\section{Introduction}

In 1984, Tarantola presented the basic idea of acoustic full waveform inversion (FWI) as a local optimization method that aims to minimize the least-squares misfit between observed and modeled seismograms. In other words, the aim of FWI is to find a subsurface model which explains the recorded seismic data (Symes 2008). Toward the end of the 80's, Mora (1987a b) and Tarantola (1986) extended the theory to the elastic case. Shortly after, Pratt and Worthington (1990) and Pratt (1990) introduced the frequency-domain version of full waveform inversion. While its high computational cost retarded its adoption for almost two decades, the advance of computing technology allowed to develop multiscale inversion, which became an area of very busy and active research, and it provided a hierarchical framework for robust inversion (Yang et al. 2015).

FWI proved to be an efficient tool for the determination of high-resolution details in multi-parameter models of complex subsurface structures, and it has been applied in different geophysical problem scales, ranging from ultrasonic data (Pratt 1999) to seismological imaging (Fichtner et al. 2009).

However, being a highly nonlinear problem, FWI techniques face other drawbacks than their elevated computational cost. They are notorious for depending strongly on the choice of a good starting model for convergence at a geologically meaningful result. Particularly, the long-wavelength components are crucial in this respect. Analyzing this dependence, Mora (1989) recognized that $\mathrm{FWI}$ has a migration component and a tomographic component. To ensure convergence of the tomographic component, a possible strategy is to start the inversion processing from the low frequencies, but this does not avoid the need of accurate initial velocity models (Biondi and Almomin, 2014).

For this reason, quite some effort has been made to come up with initial models for FWI. Traveltime tomography, Laplace-domain inversion, and migration-based velocity analysis (MVA) are some examples of seismic techniques that have been investigated for this purpose (Prieux et al. 2012).

In this paper, we investigate another possible strategy with potential to help the construction of initial velocity models for full-waveform tomography (FWT). We apply the double multi-stack technique of Schleicher and Costa (2009) to perform a time-domain MVA. This is a fully automatic tool that is useful to obtain a time migrated image and its corresponding migration velocity model in a computationally effective way. We then convert the background part of the time-migration velocity model to the depth domain by means of the time-to-depth conversion strategy based on image-wavefront propagation (Valente 2013; Valente et al. 2014; Santos et al., 2015). We evaluate the converted velocity model as an initial velocity model in an acoustic FWT process (Kurzmann et al., 2013). For comparison, we carry out the same FWT using a smoothed version of the true velocity model.

\section{Methodology}

Our workflow (Figure 1) for initial-velocity model building and FWT is built upon the following techniques.

\section{MVA by double multi-stack migration}

The workflow starts with the double multipath time migration-velocity analysis of Schleicher and Costa (2009). This MVA technique is based on the multipath-summation imaging process of Landa et al. (2006). The fundamental idea of the latter is to stack the migration results for "all possible" velocities, or at least as many models as practically reasonable. Since only "good" models yield flat events in common-image gathers, these will prevail in the overall stacked image, which thus will show the geologic structure without the need for a migration-velocity model. A weighted double stack allows to determine the associated velocity values. Below, we will refer to this technique as multi-stack migration. 


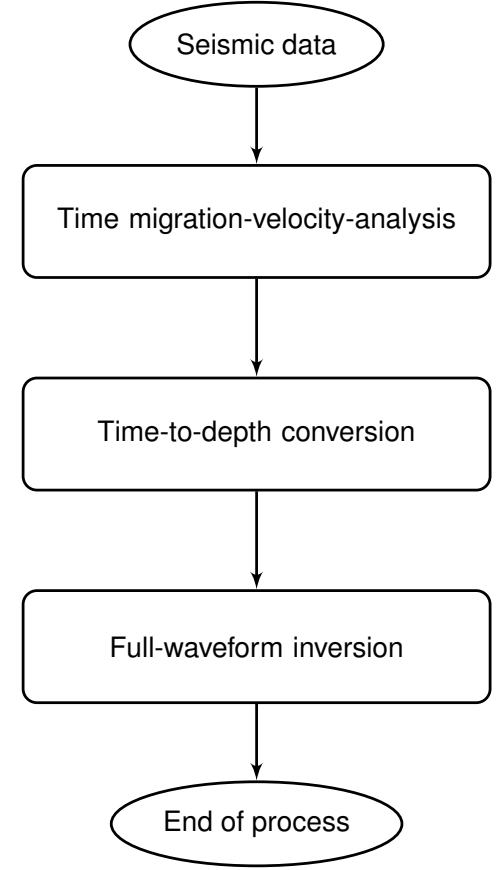

Figure 1: Velocity model building flowchart.

\section{Time-to-depth conversion and velocity estimation}

With this procedure, we can automatically construct a time-migration velocity model. However, FWI requires an initial model in depth. Therefore, we need to convert the multi-stack model to depth. For this objective, we chose the time-to-depth conversion of Valente (2013). First performance tests of that conversion procedure were reported by Valente et al. (2014).

The time-to-depth conversion of Valente (2013) is based on the algorithm of the level-set method. It pursues the construction of wavefronts instead of individual image rays. This strategy has the advantage of directly obtaining the velocity field $v(\mathbf{x})$ and the traveltime $\tau(\mathbf{x})$, avoiding to calculate the auxiliary functions $p(\mathbf{x})$ and $q(\mathbf{x})$ like in concurrent schemes (Cameron et al. 2007, 2008, Iversen and Tygel, 2008). By means of a modified fast-marching conversion algorithm, it directly determines the matrix $\gamma(\mathbf{x})$ of image-ray emergence points from the already known values of $v(\mathbf{x})$ and $\tau(\mathbf{x})$. For further details, see Valente (2013).

\section{Acoustic full waveform tomography}

To test whether the so-obtained depth model has sufficient quality for FWI, we used a modified version of the $2 \mathrm{D}$ acoustic time-domain FWT code initially implemented by Kurzmann et al. (2013). Here we briefly summarize the underlying concepts.

\section{Forward modeling}

The FWT implementation of Kurzmann et al. (2013) solves the homogeneous acoustic wave equation in the time domain by means of a time-domain finite-difference timestepping method (Alford et al., 1974) with perfectly matched

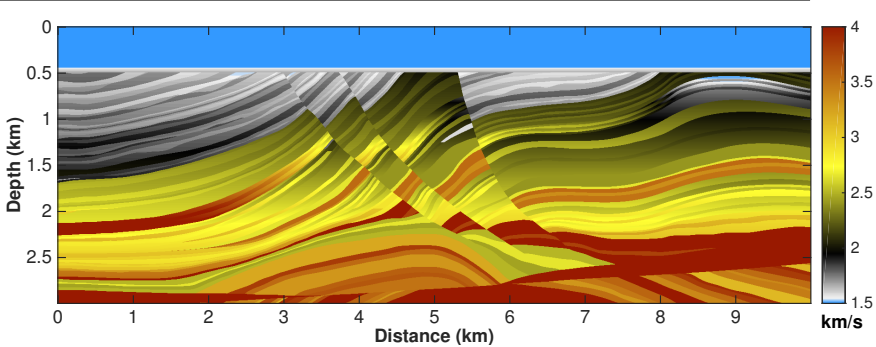

Figure 2: True velocity of the modified Marmousi-2 model.

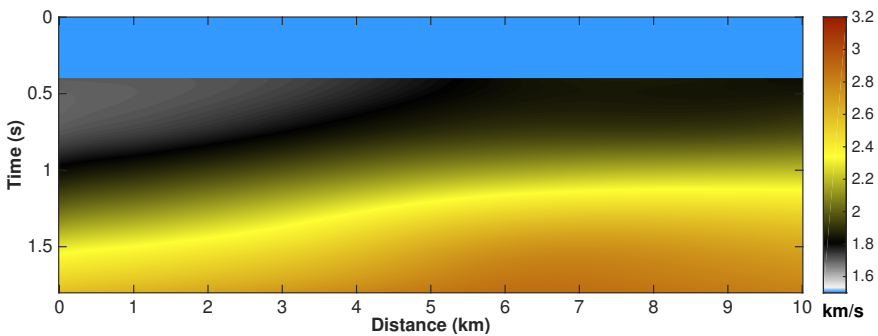

Figure 3: Time-migration velocity model obtained by the multi-stack MVA with strong regularization.

layer (PML) boundary condition (Berenger, 1994) and massive parallelization comprising domain decomposition (Bohlen 2002) and shot parallelization (Kurzmann et al. 2009). The distribution of shots on different computers provides a reduction of network traffic and consequently a speedup of the inversion algorithm.

\section{Inversion}

The objective of the code is the reconstruction of an acoustic velocity model. For simplicity, the density is considered constant and not a subject of the inversion. The solution of the inverse problem is based on the timedomain FWT of Tarantola (1984) and Mora (1987a). It comprises the adjoint method and the conjugate gradient method using a least-squares misfit function. For further details, please refer to Kurzmann et al. (2013).

\section{Numerical Example}

To test our initial-model construction, we apply it to a modified version of the 2D Marmousi model similar to the one of Kurzmann et al. (2013).

We modified a section of the Marmousi-2 model (Figure 2 based on Versteeg (1994) and Martin et al. (2002). The velocities are clipped to the range of 1500 to $4000 \mathrm{~m} / \mathrm{s}$ to reduce computational efforts. The acquisition geometry simulates a marine streamer geometry with a length of $5980 \mathrm{~m}$, consisting of 187 shots and a maximum number of 300 receivers per source. Receiver spacing was $20 \mathrm{~m}$ and the nearest offset was $45 \mathrm{~m}$. The source time function is a Ricker wavelet with peak frequency $f_{\text {peak }}=9 \mathrm{~Hz}$. The model size is $3 \mathrm{~km} \times 10 \mathrm{~km}$ which, using a grid spacing of $5 \mathrm{~m}$, resulting in a grid size of $600 \times 2000$ grid points. We set a perfectly matched layer (PML) of $150 \mathrm{~m}$ width to avoid artificial boundary reflections in finite-difference modeling. The recording time of the seismic data was $5.6 \mathrm{~s}$ with a time 


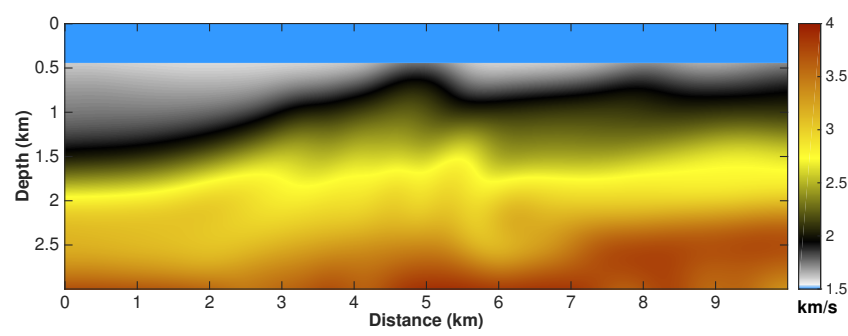

(a)

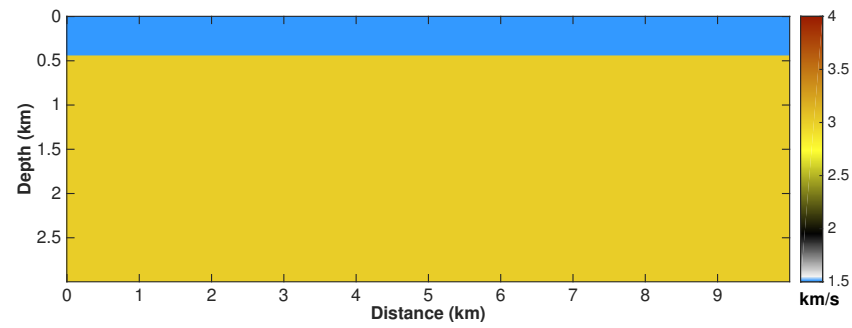

(b)

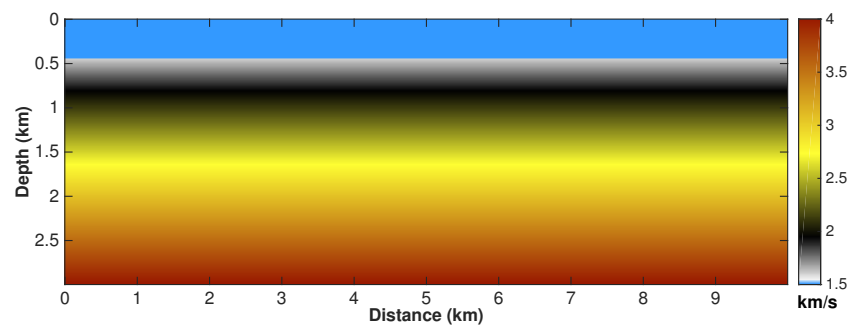

(c)

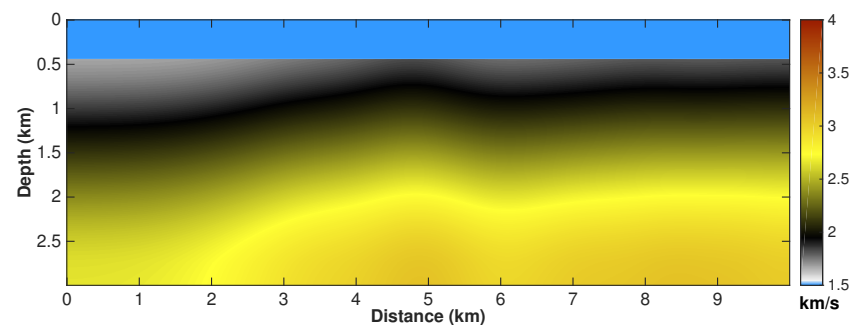

(d)

Figure 4: Initial models: (a) smoothed version obtained after low-pass filtering of the true model (Figure 2); (b) homogeneous velocity model $(v=3 \mathrm{~km} / \mathrm{s})$; (c) constant vertical gradient; (d) velocity model obtained by the double multi-stack MVA converted from time-to-depth using the image-wavefront propagation.

discretization of $7.10^{-4} \mathrm{~s}$. We chose these parameters to make the conditions for the FWT nearly ideal.

\section{Initial velocity models}

To decrease the computation time for the migration velocity analysis, and to work under more realistic conditions, we resampled the seismic data to $4 \mathrm{~ms}$ and windowed the offsets processing only half of them. From these data, we extracted a time-migration velocity model using the double multi-stack MVA with velocities between $1400 \mathrm{~m} / \mathrm{s}$ and $4200 \mathrm{~m} / \mathrm{s}$ at every $100 \mathrm{~m} / \mathrm{s}$. In order to avoid the presence of possible artifacts created during the conversion step, we used strong regularization and smoothed the time-domain

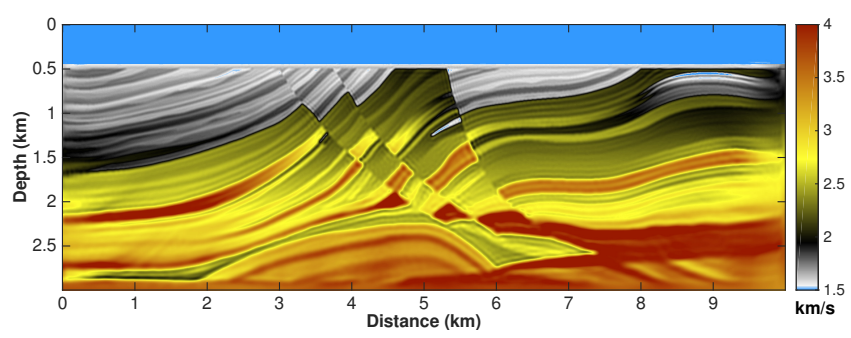

(a) 363 iterations (40353 seconds)

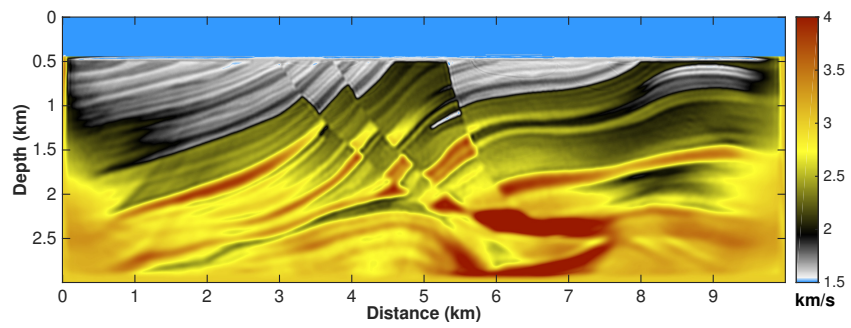

(b) 377 iterations (41726 seconds)

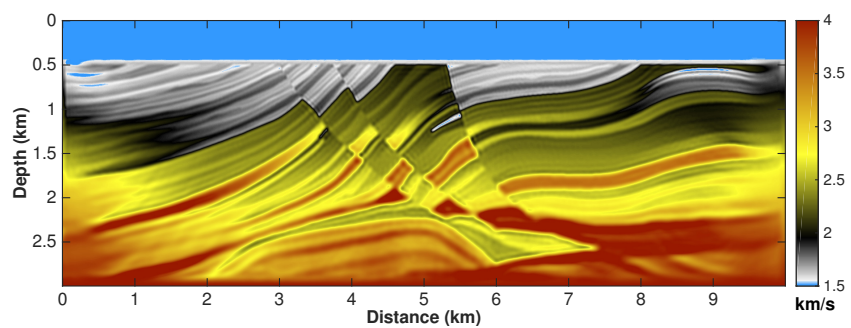

(c) 330 iterations (38485 seconds)

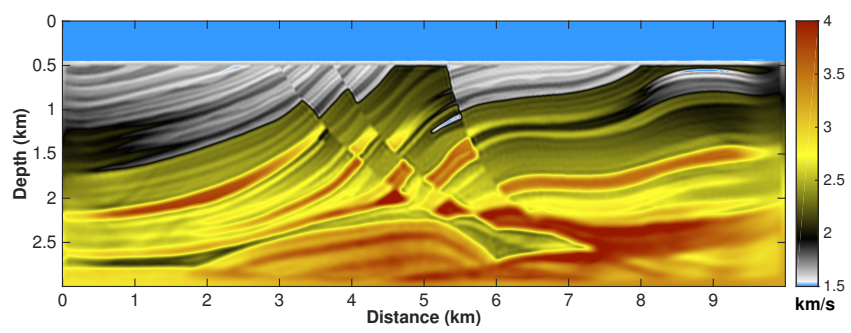

(d) 329 iterations (36081 seconds)

Figure 5: Recovered velocity models from the initial models in Figure 4 The individual amount of required iterations differs from each other.

model by one pass of a moving average with a $500 \mathrm{~m} \times$ $500 \mathrm{~m}$ (100 by 100 points) window. The resulting model is depicted in Figure 3. Less smoothed models resulted in worse FWT results. We then converted this model to depth using image-ray wavefronts as described above (Figure 4(d)).

For our evaluation, we compare the results of FWT with different initial velocity models. Figure 4(a) depicts a smooth initial velocity model, generated by application of a 2D Gaussian filter (size $1250 \mathrm{~m} \times 1250 \mathrm{~m}, \sigma=51$ ) to the sub-seafloor area of the true velocity model (Figure 2). This same $P$-wave velocity model was used by Kurzmann et al. (2013) in their sensitivity analysis of attenuation. Our other FWT tests used a constant velocity (Figure 4(b)] or a constant gradient below the see floor (Figure 4(c)). 


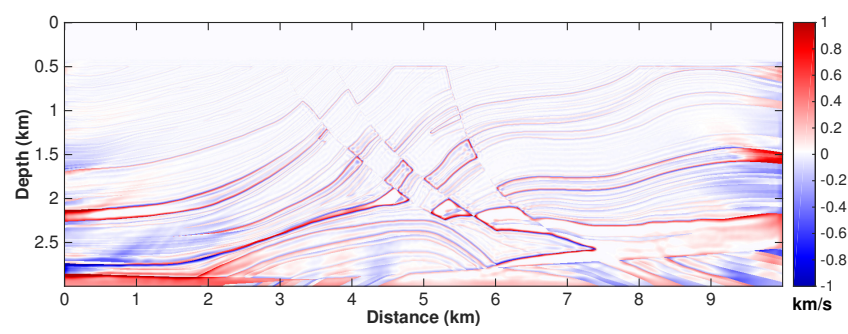

(a)

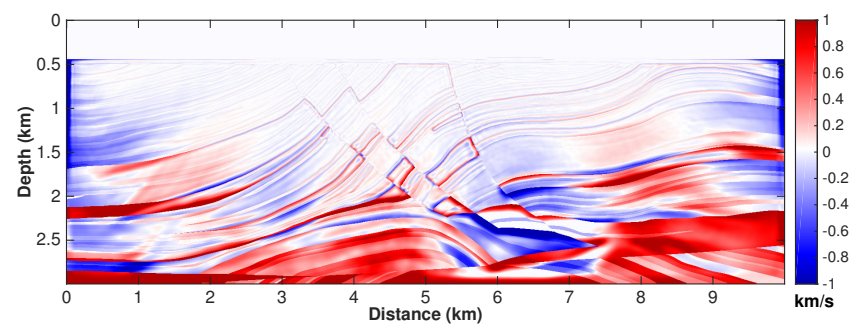

(b)

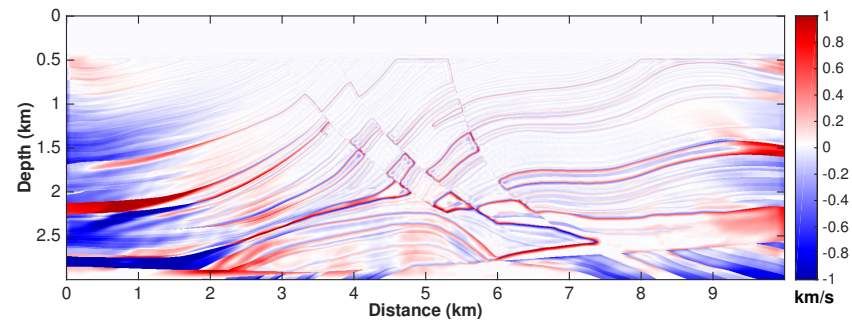

(c)

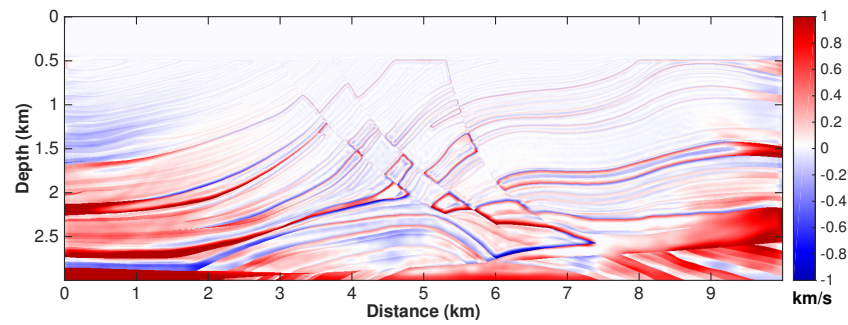

(d)

Figure 6: Differences of the results in Figure 5 to the true model in Figure 2 .

\section{FWT results}

We then executed the FWT code on the original undecimated (i.e., almost ideal) data using the starting models of Figure 4 The final inverted models, after the FWT code reached convergence at the end of the workflow, are shown in Figure 5 and their differences to the true one in Figure 6 .

We see that the results using the depth-converted multistack model (Figure $5(\mathrm{a})$ are comparable in quality to the ones obtained with the smoothed model (Figure [5(b)] or the true vertical gradient (Figure 5(c)) and clearly superior to the ones obtained with the constant starting model (Figure 5(d)). Except for the boundary region, where the data are insufficient, the model inverted when starting at the converted time model is, at some places, even superior to the one obtained from the true vertical gradient and reaches the same quality as the one obtained from the smoothed model (compare difference images in Figure 6).

\section{Conclusion}

In this work, we have presented a workflow for the construction of initial velocity-models for FWT methods. In an attempt to aid the search for more efficient modelbuilding tools, we investigate the applicability of a recent automatic time MVA method. This method stacks twice over migrated images for many models with different weights in order to extract stationary migration velocities from the ratio of the images. Thus, it is able to generate a velocity model and a time-migrated image without a priori information.

For the use of such a velocity model in FWT methods, the result needs to be converted from time to depth. For this purpose, we chose a strategy based only on image-ray wavefront propagation. The results confirmed the method's efficiency in very complex geology structures, i.e., models with strong velocity variations.

Our first numerical results indicate the potential of using a combination of these methods to achieve a fully automatic tool for initial-model building in an FWT workflow. In our tests, the method was able to produce a sufficiently accurate initial model for an FWT under nearly ideal conditions converges to a model of comparable quality as when starting at a smoothed version of the true model. This indicates that correct background velocity information can be successfully extracted from automatic time-domain migration velocity analysis even in media where timemigration cannot provide satisfactory seismic images.

Future investigations will have to show whether some model detail can be already introduced in the time domain or added in an additional depth-domain MVA step in order to reduce the number of necessary FWT iterations and if the inversion can still be successful from such initial models if the data are less than ideal.

\section{Acknowledgments}

This research was kindly supported by the Brazilian national research agencies CAPES, CNPq, FAPESP, and FINEP. Henrique B. Santos is grateful to CGG-Brazil, Petrobras, ANP and PRH-PB230 for his fellowships. Furthermore, we are grateful for using the supercomputers JUROPA at Jülich Supercomputing Centre and ForHLR at Steinbuch Centre for Computing (KIT, Karlsruhe; funded by the state of Baden-Württemberg and the federal government of Germany). Additional support for the authors was provided by the sponsors of the Wave Inversion Technology (WIT) Consortium. Past WIT Reports are available at www.wit-consortium.de.

\section{References}

Alford, R. M., K. R. Kelly, and D. M. Boore, 1974, Accuracy of finite-difference modeling of the acoustic wave equation: Geophysics, 39, 834-842, doi: 10.1190/1.1440470.

Berenger, J. P., 1994, A perfectly matched layer for the absorption of electromagnetic waves: Journal of Computational Physics, 114, 185-200, doi: 10.1006/jcph.1994.1159.

Biondi, B., and A. Almomin, 2014, Simultaneous inversion 
of full data bandwidth by tomographic full-waveform inversion: Geophysics, 79, WA129-WA140, doi: 10.1190/geo2013-0340.1.

Bohlen, T., 2002, Parallel 3D viscoelastic finite difference seismic modelling: Computers \& Geosciences, 28, 887899, doi: 10.1016/s0098-3004(02)00006-7.

Cameron, M. K., S. B. Fomel, and J. A. Sethian, 2007, Seismic velocity estimation from time migration: Inverse Problems, 23, 1329-1369.

- 2008, Time-to-depth conversion and seismic velocity estimation using time-migration velocity: Geophysics, 73, VE205-VE210, doi: 10.1190/1.2967501.

Fichtner, A., B. L. N. Kennett, H. Igel, and H.-P. Bunge, 2009, Full seismic waveform tomography for uppermantle structure in the Australasian region using adjoint methods: Geophysical Journal International, 179, 17031725, doi: 10.1111/j.1365-246X.2009.04368.x.

Iversen, E., and M. Tygel, 2008, Image-ray tracing for joint 3D seismic velocity estimation and time-to-depth conversion: Geophysics, 73, S99-S114, doi: doi: 10.1190/1.2907736.

Kurzmann, A., D. Köhn, A. Przebindowska, N. Nguyen, and T. Bohlen, 2009, 2D Acoustic Full Waveform Tomography: Performance and Optimization: Presented at the Extended Abstracts, 71st Conference and Technical Exhibition, EAGE.

Kurzmann, A., A. Przebindowska, D. Kohn, and T. Bohlen, 2013, Acoustic full waveform tomography in the presence of attenuation: a sensitivity analysis: Geophysical Journal International, 195, 985-1000, doi: 10.1093/gji/ggt305.

Landa, E., S. Fomel, and T. J. Moser, 2006, Path-integral seismic imaging: Geophysical Prospecting, 54, 491503, doi: 10.1111/j.1365-2478.2006.00552.x.

Martin, G. S., K. J. Marfurt, and S. Larsen, 2002, Marmousi-2: An updated model for the investigation of AVO in structurally complex areas: SEG Technical Program Expanded Abstracts, 72 Annual International Meeting, SEG, 1979-1982.

Mora, P., 1987a, Elastic inversion of field data: SEP report, $51,211-218$.

, 1987b, Nonlinear two-dimensional elastic inversion of multioffset seismic data: Geophysics, 52, 1211-1228, doi: 10.1190/1.1442384.

1989, Inversion= migration+ tomography: Geophysics, 54, 1575-1586, doi: 10.1190/1.1442625.

Pratt, R. G., 1990, Inverse theory applied to multi-source cross-hole tomography. Part 2: elastic wave-equation method: Geophysical Prospecting, 38, 311-329.

_ 1999 , Seismic waveform inversion in the frequency domain, Part 1: Theory and verification in a physical scale model: Geophysics, 64, 888-901.

Pratt, R. G., and M. H. Worthington, 1990, Inverse theory applied to multi-source cross-hole tomography. Part 1: acoustic wave-equation method: Geophysical Prospecting, 38, 287-310, doi: 10.1111/j.13652478.1990.tb01847.x.

Prieux, V., G. Lambaré, S. Operto, and J. Virieux, 2012, Building starting models for full waveform inversion from wide-aperture data by stereotomography: Geophysical Prospecting, 61, 109-137, doi: 10.1111/j.13652478.2012.01099.x.

Santos, H. B., L. S. S. Valente, J. C. Costa, and J. Schleicher, 2015, Time-to-depth conversion and velocity estimation by wavefront-propagation: SEG International Exposition and 85th Annual Meeting, doi: 10.1190/segam2015-5922300.1.

Schleicher, J., and J. C. Costa, 2009, Migration velocity analysis by double path-integral migration: Geophysics, 74, WCA225-WCA231, doi: 10.1190/1.3162482.

Symes, W. W., 2008, Migration velocity analysis and waveform inversion: Geophysical Prospecting, 56, 765790, doi: 10.1111/j.1365-2478.2008.00698.x.

Tarantola, A., 1984, Inversion of seismic reflection data in the acoustic approximation: Geophysics, 49, 12591266, doi: 10.1190/1.1441754.

_ 1986, A strategy for nonlinear elastic inversion of seismic reflection data: Geophysics, 51, 1893-1903, doi: P10.1190/1.1442046.

Valente, L. S. S., 2013, Evaluation of algorithms for the conversion of velocity models from time to depth: Master's thesis, Federal University of Pará. (In Portuguese).

Valente, L. S. S., H. B. Santos, J. C. Costa, and J. Schleicher, 2014, A strategy for time-to-depth conversion and velocity estimation: Annual WIT Report, 18, 137-151.

Versteeg, R., 1994, The Marmousi experience: Velocity model determination on a synthetic complex data set: The Leading Edge, 13, 927-936, doi: 10.1190/1.1437051.

Yang, P., J. Gao, and B. Wang, 2015, A graphics processing unit implementation of time-domain fullwaveform inversion: Geophysics, 80, F31-F39, doi: 10.1190/geo2014-0283.1. 